\title{
Spectra and field distribution of photonic-crystal structure with inclusions of metal nanoparticles
}

\author{
I.A. Glukhov ${ }^{1}$, S.G. Moiseev ${ }^{1,2}$ \\ ${ }^{1}$ Ulyanovsk State University, 42 Lev Tolstoy Str., 432017, Ulyanovsk, Russia \\ ${ }^{2}$ Kotelnikov Institute of Radio Engineering and Electronics of the Russian Academy of Sciences, Ulyanovsk Branch, $48 / 2$ Goncharov Str., 432011 \\ Ulyanovsk, Russia
}

\begin{abstract}
Transmittance and reflectance spectra as well as field distribution in 1D photonic-crystal structure with embedded dielectric layer and monolayer of metal nanoparticles are characterized. The influence of plasmonic monolayer location on defect modes of photonic-crystal structure is demonstrated with respect to domains of field confinement in the cavity area.

Keywords: nanoplasmonics; photonic-crystal structure; defect mode; field localization

\section{Introduction}

In recent years 1D photonic-crystal structures (PCS) created on the basis of different materials are of a special interest to researchers. Owing to periodic modulation of refractive index, photonic spectrum of these structures has a band gap, in which incident radiation is practically totally reflected. This property is critical for practical use as it enables to control optical radiation in data-transmission systems and in laser technology. Particularly remarkable is Fabry-Perot microresonator-like structure composed of two Bragg reflectors with defect layer there between. Defect layer in such-like structure plays the role of optical microcavity (microresonator) on which electromagnetic radiation can be localized. This can add to material-radiation interaction effects.

Varying geometrical and physical properties of the structure it is possible to control spectral characteristics of PCS $[1,2]$ that enables to improve considerably their functionality. For example, through breakdown of the structure periodicity or using materials with controlled properties (non-linear, resonant, magnetogirotropic) photonic spectrum of PCS can be modified considerably. Metallic-dielectric nanocomposite media are advanced materials to be used as microcavity of photonic-crystal resonator. In the field of plasmonic resonance vigorous dispersion of optical properties of these materials is observed [3,4]. This paper describes the case of ultrathin resonance structure as a monolayer of metal nanoparticles, plasmonic frequency of which coincides with defect mode frequency of PCS.
\end{abstract}

\section{PCS material parameters and transfer matrixes}

In order to calculate reflectivity and transmission of plane-layered structure with embedded monolayer of nanoparticles we employ T-matrix technique. A special case is interface, optical qualities of which are determined by Fresnel reflection and transmission coefficients [5]. Since array of nanoparticles situated in the same plane interacts with electromagnetic wave like plane interface, it can be also treated as an interface with its own reflection and transmission coefficients.

We assume that there are $\mathrm{N}$ interfaces in the layered medium, and they are formed by N-1 interfacial boundaries and a single layer of nanoparticles. A space between interfaces is packed by media with different refraction indexes $n_{i}(i=0 \ldots N)$. Semi-infinite media are those that have $n_{0}$ and $n_{N}$ refraction indexes. Let a harmonic wave is incident on a layered structure in z-direction. To describe its propagation in PCS we introduce the following notation for electric field components inside structure: $E_{i}\left(z_{i}^{-}\right)$to the left of i number interface; $E_{i}\left(z_{i}^{+}\right)$to the right of i number interface; $E_{f}$ for the propagating forward wave; $E_{b}$ for the propagating backward wave.

According to the introduced notation, complex amplitudes of counter-propagating waves on $m$ interface in the layer with reflection index $n_{m-1}$ are equal to $E_{f}\left(z_{m}^{-}\right)$and $E_{b}\left(z_{m}^{-}\right)$. At the same interface but in the layer with reflection index $n_{m}$ they are equal to $E_{f}\left(z_{m}^{+}\right)$and $E_{b}\left(z_{m}^{+}\right)$. Relationship of these fields on m-interface (to the left and to the right of it) can be expressed as matrix equation:

$$
\begin{aligned}
& \left(\begin{array}{c}
E_{f}\left(z_{m}^{-}\right) \\
E_{b}\left(z_{m}^{-}\right)
\end{array}\right)=I_{m-1, m}\left(\begin{array}{c}
E_{f}\left(z_{m}^{+}\right) \\
E_{b}\left(z_{m}^{+}\right)
\end{array}\right), \\
& I_{m-1, m}=\frac{1}{t_{m-1, m}}\left(\begin{array}{cc}
1 & -r_{m, m-1} \\
r_{m-1, m} & t_{m-1, m} t_{m, m-1}-r_{m-1, m} r_{m, m-1}
\end{array}\right),
\end{aligned}
$$


where $r_{i, j}, t_{i, j}$ are complex reflection and transmission coefficients of the interface deviding media with refrection indexes $n_{i}$ and $n_{j}$ when the lightwave is incident from the medium with refraction index $n_{i}$. In case of plane interface $r_{i, j}$ and $t_{i, j}$ are Fresnel coefficients [5]. Relationship of the fields on two interfaces of $m$ and $m+1$ numbers confining homogeneous layer of $m$ number is via transfer matrix $\hat{F}_{m}$ :

$$
\begin{aligned}
& \left(\begin{array}{l}
E_{f}\left(z_{m}^{-}\right) \\
E_{b}\left(z_{m}^{-}\right)
\end{array}\right)=\hat{F}_{m}\left(\begin{array}{l}
E_{f}\left(z_{m}^{+}\right) \\
E_{b}\left(z_{m}^{+}\right)
\end{array}\right), \\
& \hat{F}_{m}=\left(\begin{array}{ll}
\exp \left(-i \delta_{m}\right) & 0 \\
0 & \exp \left(i \delta_{m}\right)
\end{array}\right),
\end{aligned}
$$

where $\delta_{m}=k n_{m} L_{m}$ is phase thickness of the layer; $k=\omega / c$ is the wave number.

Applying expressions (1) - (4) to the entire PCS we obtain relation for the amplitudes to the left of the first interface and to the right of the last (with number $N$ ) interface:

$$
\begin{aligned}
& \left(\begin{array}{c}
E_{f}\left(z_{1}^{-}\right) \\
E_{b}\left(z_{1}^{-}\right)
\end{array}\right)=\hat{G}\left(\begin{array}{c}
E_{f}\left(z_{N}^{+}\right) \\
E_{b}\left(z_{N}^{+}\right)
\end{array}\right), \\
& \hat{G}=I_{0,1} F_{1} I_{1,2} F_{2} \quad F_{N-1} I_{N-1, N} .
\end{aligned}
$$

Note that in semi-infinite medium with refraction index $n_{N}$ there exists only transmitted wave, therefore we shall assume $E_{b}\left(z_{N}^{+}\right)=0$ in (5).

Reflectance and transmittance of PCS are calculated from the formulas $T=\left|\frac{1}{\hat{G}_{11}}\right|^{2}, \quad R=\left|\frac{\hat{G}_{21}}{\hat{G}_{11}}\right|^{2}$.

To calculate field distribution in PCS we can use expressions similar to (5), in the left part of which column elements are substituted with amplitudes of local fields in the corresponding points.

\section{Reflection and transmission spectra of nanoparticle monolayer}

For the analysis of the properties of PCS with embedded nanocomposite monolayer film of nanoparticles placed into dielectric matrix, it is necessary to know amplitude coefficients of monolayer reflection and refraction. Analytical calculation of these coefficients is not a trivial task; therefore we use numerical technique - FEM implemented in COMSOL Multiphysics software. For simplicity we consider the case of the ordered monolayer film in which nanoparticles are located at the sites of square lattice lying in the palne $(x y)$.

Taking into account structure symmetry, we took fourth of the structure's unit cell (Fig. 1). Such domain contains fourth of nanoparticle and is of the size equal to $1 / 2$ period of the structure along the direction of $x$ and $y$ coordinate axes. In order to obtain the entire monolayer it is necessary to apply reflection operations to the domain shown in Fig. 1 (to complete the unit to the full with a spherical shape particle), and then to apply transmission operations along $x$ and $y$ coordinate axes. The structure thus obtained will be 2D array of nanoparticles in (xy) plane. Boundary conditions are selected in such a way that the model fits the case of normal (to the plane of monolayer film) incidence of the light wave. Incident polarization is oriented parallel to one of the crystal axes of monolayer film.

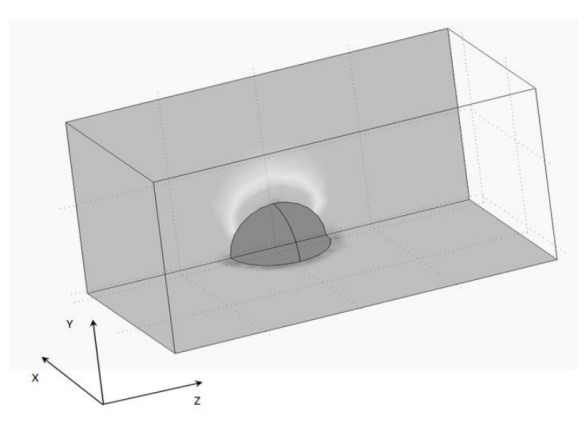

Fig. 1. Modeling domain in Comsol Multiphysics software. 
As a medium in which nanoparticle monolayer is weighted, we use material with dielectric constant $\varepsilon_{m}=2.25$. To calculate dielectric constant of metal nanoparticles we use relation of Drude theory [5]:

$\varepsilon_{p}(\omega)=\varepsilon_{0}-\frac{\omega_{p}^{2}}{\omega^{2}+i \omega \gamma}$

where $\omega_{p}$ is plasmonic frequency, $\varepsilon_{0}$ is lattice contribution, $\gamma$ is relaxation parameter. For definiteness, as nanoparticle material we use silver, for which $\omega_{p}=1.36 \cdot 10^{16} \mathrm{c}^{-1}, \varepsilon_{0}=5, \gamma=3 \cdot 10^{13} \mathrm{c}^{-1}$.

Modeling outcomes for optical properties of silver nanoparticle monolayer are shown in Fig. 2. It is obvious that in the domain of surface plasmonic resonance of nanoparticles (resonant wavelength falls within $435 \mathrm{~nm}$ ) monolayer film reflection and transmission spectra are subject to strong changes. Amplitudes of the observed values in resonance region depend on the surface concentration of nanoparticles: with decrease in the average distance among nanoparticles resonance becomes more pronounced; the width of resonance increases and frequency shift of resonance towards short wavelength region is observed. Thus, spectral characteristics of the monolayer of metal nanoparticles depend on internal geometrical parameters which make it possible to control to a certain extent its influence on spectrum of PCS.

\section{Analysis of the properties of photonic-crystal structure with monolayer of nanoparticles}

Let us consider PCS, in which between two dielectric reflectors there is a defect layer consisting of dielectric matrix and a monolayer film of nanoparticles. Transfer matrix of such structure can be expressed as:

$N=I_{0,1} F_{1} \quad I_{d-1, d} F_{d 1} F_{s} F_{d 2} I_{d, d+1} \quad F_{N-1} I_{N-1, N}=M^{a} D M^{b}$,

where $F_{s}$ is transfer matrix of nanoparticle monolayer; $F_{d 1}$ and $F_{d 2}$ are transfer matrixes of the layers that edge monolayer of nanoparticles; $M^{a}$ and $M^{b}$ are transfer matrixes that describe dielectric reflectors containing $a$ and $b$ binary layers respectively. Binary layers of dielectric reflectors consist of two layers of isotropic dielectric material of real transmissivities $\varepsilon_{j}$ and thickness $L_{j}(j=1,2)$. For modeling optical properties of the structure we assumed $\varepsilon_{1}=6.25$ and $\varepsilon_{2}=2.25$. Thickness of layers of the structure meets the requirements $L_{1,2}=\lambda_{0} / 4 \sqrt{\varepsilon_{1,2}}$; thickness of defect layer is equal to $L_{d}=\lambda_{0} / \sqrt{\varepsilon_{d}}$, where $\lambda_{0}$ - wavelength in vacuum calculated for the central frequency of the photonic band gap. Presence of defect layer in PCS leads to the occurrence in the photonic band gap of narrow spectral transmission band with the peak value of transmission index which is close to 1 .

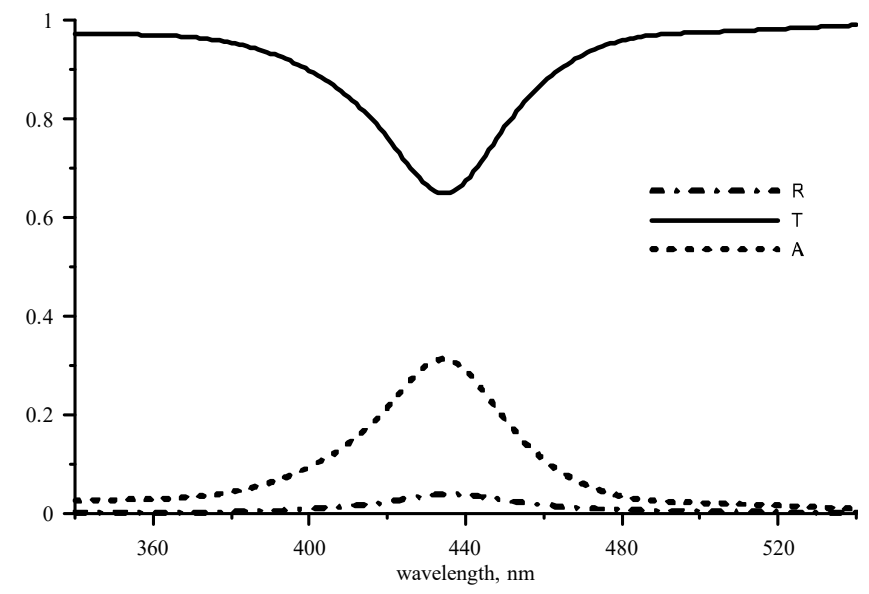

Fig. 2. Reflection, transmission and absorption spectra for monolayer of silver nanoparticles. Period of structure is $10 \mathrm{~nm}$.

Figures 3 and 4 show field distribution (square amplitude of dielectric field intensity) and transmission and reflection spectra of PCS of $M^{5} D \bar{M}^{5}$-type depending on location of the monolayer of plasmonic nanoparticles. Location of the monolayer is given by relations $L_{d 1}=L_{d} / 2+\Delta, L_{d 2}=L_{d} / 2-\Delta$, where $L_{d}$ is thickness of the central layer, which is divided by a monolayer of nanoparticles into two domains of the thickness $L_{d 1}$ and $L_{d 2}$ respectively. The domain of the thickness $L_{d 1}$ is located from incidence of external electromagnetic wave. Thus, $\Delta$ is a value of monolayer film displacement from the center of PCS. Parameters of dielectric layers of PCS correlate such that in the center of PCS, i. e. for $\Delta=0$, field amplitude is close to zero (standing-wave nod), and in displacement by $\Delta= \pm 71 \mathrm{~nm}$ the amplitude reaches a maximum value (antinode of standing wave). The given relations indicate that if monolayer film of nanoparticles is located in the center $(\Delta=0)$, field distribution and transmission and reflection spectra are practically comparable with the case of monolayer-free PCS. Such peculiarity is explained practically by total absence of electrodynamic interaction between monolayer film and lightwave in standing-wave node. Monolayer film is really in the domain of electromagnetic shadow. If nanoparticles are in the domain of strong field $(\Delta=71 \mathrm{~nm})$, when intensity 
of electromagnetic scattering by monolayer is the highest, defect mode transformation is observed. Transformation is displayed as decrease in its amplitude and splitting of spectrum curves.

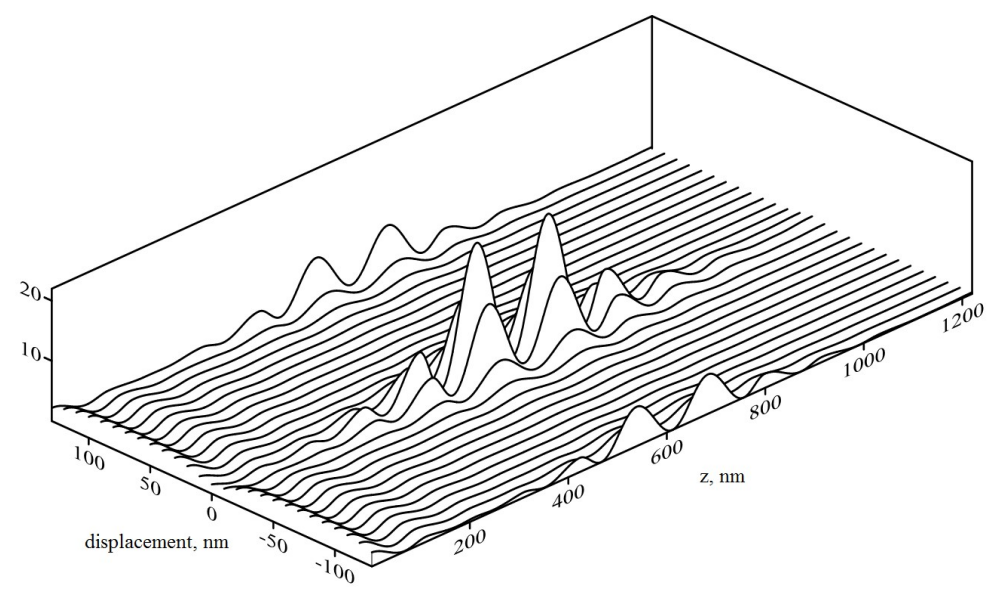

Fig. 3. The distribution of electromagnetic field amplitude (in relative units) throughout PCS as a function of the value of displacement of monolayer of nanoparticles from the center of the structure.

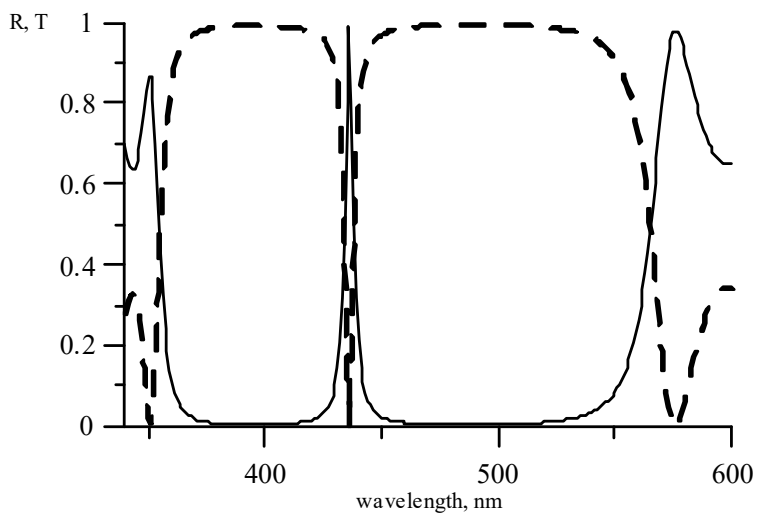

(a)

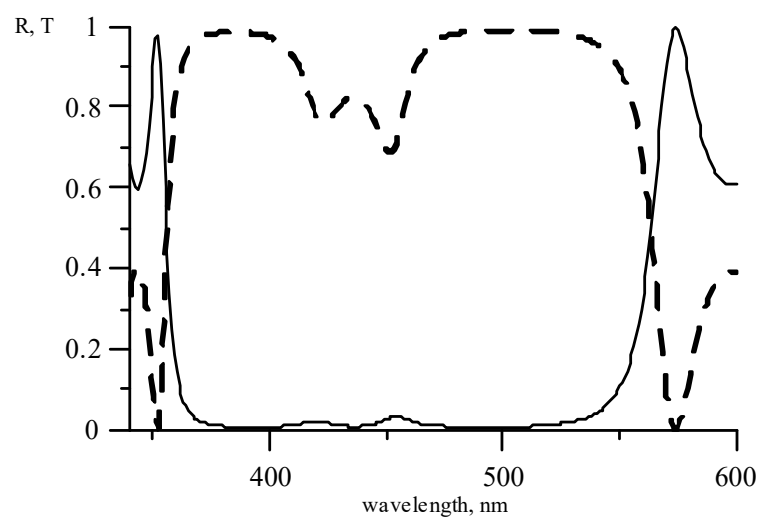

(b)

Fig. 4. Ttransmission (dashed line) and reflection (continuous line) spectra of PCS when monolayer displacement is (a) $\Delta=71 \mathrm{~nm}$, (b) $\Delta=0$.

\section{Conclusions}

We have demonstrated that control of transmission and reflection coefficients corresponding to defect mode in photonic band gap of PCS is capable owing to the use of nanoparticle monolayer with plasmonic resonance. It is shown that defect mode amplitude is heavily dependent on the location of monolayer. Dependence of spectral characteristics of the layered structure on the location of plasmonic monolayer is attributed to the inhomogeneity of electromagnetic field distribution in the optical microcavity placed between distributed Bragg reflectors.

\section{Acknowledgements}

This work was supported by the Ministry of Education and Science of the Russian Federation (State Contracts Nos. 3.5698.2017/P220 and 3.8388.2017/ITR) and the Russian Foundation for Basic Research (Projects Nos. 15-07-08111 and 17-02-01382). 


\section{References}

[1] Vorobev LE, Ivchenko EL, Firsov DA, Shalygin VA. Optical properties of nanostructures. Saint Petersburg: "Nauka" Publisher 2001.

[2] Gaponenko SV, Rosanov NN, Ivchenko EL. Optics of nanostructures. Saint Petersburg: "Nedra" 2005.

[3] Moiseev SG, Ostatochnikov VA. Defect modes of one-dimensional photonic-crystal structure with a resonance nanocomposite layer. Quantum Electron 2016; 46(8): 743-748.

[4] Vetrov SY, Avdeeva AY, Timofeev IV. Spectral properties of a one-dimensional photonic crystal with a resonant defect nanocomposite layer. JETP 2011; 113(5): 755-761.

[5] Born M, Wolf E. Principles of Optics. Cambridge: Cambridge University Press 1999. 